\title{
Comparison of percutaneous cannulated screw fixation and calcium sulfate cement grafting versus minimally invasive sinus tarsi approach and plate fixation for displaced intra-articular calcaneal fractures: a prospective randomized controlled trial
}

Yongzeng Feng, Xiaolong Shui, Jianshun Wang, Leyi Cai, Yang Yu, Xiaozhou Ying, Jianzhong Kong and Jianjun Hong ${ }^{*}$

\begin{abstract}
Background: The management of displaced intra-articular calcaneal fractures (DIACFs) remains challenging and controversial. A prospective randomized controlled trial was conducted to compare percutaneous reduction, cannulated screw fixation and calcium sulfate cement (PR+CSC) grafting with minimally invasive sinus tarsi approach and plate fixation (MISTA) for treatment of DIACFs.
\end{abstract}

Methods: Ultimately, 80 patients with a DIACFs were randomly allocated to receive either PR+CSC $(N=42)$ or MISTA ( $N=38$ ). Functional outcomes were evaluated using the American Orthopaedic Foot and Ankle Society (AOFAS) hindfoot scores. Radiological results were assessed using plain radiographs and computed tomography (CT) scans, and postoperative wound-related complications were also recorded.

Results: The average time from initial injury to operation and the average operation time in the PR+CSC group were both significantly shorter than those in the MISTA group $(p<0.05)$. There were significantly fewer complications in the PR+CSC group than those in the MISTA group (7.1\% vs $28.9 \%, p<0.001)$. The calcaneal width immediate postoperatively and at the final follow-up in the MISTA group were obviously improved compared to those in the PR+CSC group $(p<0.001)$. The variables of sagittal motion and hindfoot motion of the AOFAS scoring system in the PR+CSC group were significantly higher than those in the MISTA group $(p<0.05)$. The good and excellent results in the two groups were comparable for Sanders Type-II calcaneal fractures, but the good to excellent rate in the MISTA group was significantly higher for Sanders Type-III fractures $(p<0.05)$.

(Continued on next page)

\footnotetext{
* Correspondence: hongjianjun_fey@163.com

Department of Orthopaedics Surgery, The Second Affiliated Hospital of

Wenzhou Medical University, NO. 109, Xue Yuan West Road, Lucheng District,

Wenzhou, Zhejiang Province 325027, China
}

C 2016 The Author(s). Open Access This article is distributed under the terms of the Creative Commons Attribution 4.0 International License (http://creativecommons.org/licenses/by/4.0/, which permits unrestricted use, distribution, and reproduction in any medium, provided you give appropriate credit to the original author(s) and the source, provide a link to the Creative Commons license, and indicate if changes were made. The Creative Commons Public Domain Dedication waiver (http://creativecommons.org/publicdomain/zero/1.0/) applies to the data made available in this article, unless otherwise stated. 
(Continued from previous page)

Conclusion: The clinical outcomes are comparable between the two minimally invasive techniques in the treatment of Sanders Type-II DIACFs. The PR+CSC grafting is superior to the MISTA in terms of the average time between initial injury and operation, operation time, wound-related complications and subtalar joint activity. However, the MISTA has its own advantages in improving the calcaneal width, providing a more clear visualization and accurate reduction of the articular surface, especially for Sanders Type-III DIACFs.

Trial registration: ChiCTRIOR16008512. 21 May 2016.

Keywords: Calcaneus, Intra-articular fractures, Minimally invasive, Percutaneous fixation, Sinus tarsi approach, Randomized controlled trial

\section{Background}

The management of displaced intra-articular calcaneal fractures (DIACFs) remains challenging and controversial [1]. Open reduction and internal fixation (ORIF) through an extensile lateral approach has been widely accepted and established as a standard treatment for DIACFs [2, 3]. However, a fairly high wound-related complication rate has been reported with this approach, including wound edge necrosis, dehiscence, hematoma, infection and injury to the sural nerve [4-7].

In an attempt to lower the complication rate, various minimally invasive techniques have recently been introduced, including external fixation, percutaneous fixation, arthroscopically assisted fixation, and minimal incision techniques via medial, modified lateral (such as the sinus tarsi approach), longitudinal, or combined approaches [8-14]. These techniques have been reported as effective in minimizing soft tissue trauma, thereby reducing the incidence of woundrelated complications.

As one of the most popular and effective minimally invasive techniques, the sinus tarsi approach not only can fully expose the posterior facet and the anterolateral fragment, but it also significantly reduces the incidence of postoperative wound complications [11, 12, 15]. In order to further shorten the operative time and decrease the wound complication rate, we introduced, in 2006, percutaneous reduction, cannulated screw fixation and calcium sulfate cement (CSC) grafting. A preliminary clinical study carried out by our team found that compared with the traditional L-shaped extensile lateral approach, this new technique allowed earlier weight-bearing, reduced subtalar joint stiffness and a reduced wound complication rate, while improving the patients' satisfaction [16]. The purpose of the present study was to compare the functional and radiological outcomes and complications of our percutaneous reduction, cannulated screw fixation and CSC grafting with those of the minimally invasive sinus tarsi approach and plate fixation for treatment of DIACFs.

\section{Methods \\ Subjects}

Patients with a Sanders Type-II or Type-III calcaneal fracture, who were the consecutive candidates for surgical treatment at our hospital from January 2009 to December 2011, were randomly allocated to either the percutaneous reduction, cannulated screw fixation and CSC grafting (the $\mathrm{PR}+\mathrm{CSC}$ group) or the minimally invasive sinus tarsi approach and plate fixation (the MISTA group). The patients were randomly divided into the two groups using coin tossing method. Three senior surgeons were randomly assigned to each group to perform the surgeries using either surgical method.

The inclusion criteria were: 1) unilateral Sanders Type-II or Type-III intra-articular calcaneal fracture; 2 ) the age was greater than or equal to 18 years old; and 3) closed fracture. The exclusion criteria were: 1) severe medical ailments (severe vascular or neurologic injury, diabetes) or contraindications (known local or systemic infection); 2) Sanders Type-IV, bilateral or open calcaneal fractures; 3 ) severe and polytraumatic injuries or polytrauma of the ipsilateral lower limb when admitted; and 4) refused to accept the treatment plan. All patients agreed to participate in this clinical trial by signing an informed consent form. The study was approved by the Ethical Board Review of the Second Affiliated Hospital of Wenzhou Medical University (Wenzhou, Zhejiang, China), and was performed in accordance with the ethical standards of the Declaration of Helsinki of 1964.

\section{Preoperative management}

All patients were evaluated using preoperative calcaneal radiographs and computed tomography (CT) scans and two-dimensional reconstruction of the injured foot. The swelling of the hindfoot did not need to subside if the patients were randomly assigned to the $\mathrm{PR}+\mathrm{CSC}$ group. All surgeries were performed with patients placed in the lateral decubitus position under either epidural or spinal anesthesia. Tourniquets were applied routinely. 


\section{Surgical techniques}

As previously described, the percutaneous reduction, cannulated screw fixation and CSC grafting procedures were briefly summarized as below [16]. First, we crossed the tuberosity with a $6.5-\mathrm{mm}$ Schanz pin via a stab incision to reduce the height and length of the calcaneus. Then, another 6.5-mm Schanz pin was introduced into the fragment with the displaced posterior facet and levered the compressed facet under fluoroscopic guidance. When necessary, especially in Sanders Type-III fractures, another Schanz pin was introduced percutaneously through the lateral cortex of the calcaneus to push up any remaining depressed parts of the subtalar joint surface. Once the Böhler's angle and articular surface were reduced, two Kirschner wires were inserted from the lateral side to the sustentaculum to sustain the reduced joint surface. Then another two Kirschner wires were introduced from the tuberosity to the anterior part of the calcaneus in different directions to fix the primary and secondary fracture line. After the closed reduction and provisional fixation were performed, the Kirschner wires were replaced by 6.5 - and $3.5-\mathrm{mm}$ cannulated screws percutaneously. Finally, the CSC (Wright Medical Technology, Arlington, TN) was then slowly and carefully injected into the bone void in the body created after the reduction under fluoroscopic guidance (Fig. 1).

The standard minimally invasive sinus tarsi approach was performed as follows [12]: The front incision was directed toward the lateral wall bone of the anterior process of the calcaneus. Using sharp dissection, this incision was made down to the posterior subtalar joint capsule. After moderate exposure of the lateral wall of the calcaneus, we elevated the subtalar articular surface with a dedicated elevator inserted into the fracture and then a $3.5 \mathrm{~mm}$ Steinmann pin was inserted into the calcaneus tuberosity followed by longitudinally extending it to the posterior articular surface to correct the Böhler angle. After reduction, the Steinmann pin was driven into the anterior process of the calcaneus. After the calcaneus length, height and width, and Böhlers and Gissanes angles were recovered satisfactorily under Carm fluoroscopy, the plate was inserted through the incision. Following the plate insertion, the screws were well fixed through the incision or percutaneously under C-arm fluoroscopy (Fig. 2).

\section{Postoperative management}

All patients in both groups underwent the same postoperative management protocol. The patients were encouraged to do non-weight-bearing exercises including extension and plantar flexion as early as the pain could be endured and use crutch while walking two or three days postoperatively. Partial weight-bearing was permitted at four weeks post-operation and then progressed gradually. Full weight-bearing was not allowed until bony union confirmed on radiographs, which was around three months postoperatively. Each patient received follow-up at 6 weeks, 3, 6, and 12 months post-operation, and then yearly thereafter.

\section{Clinical evaluation}

The functional outcomes were assessed using the American Orthopaedic Foot and Ankle Society (AOFAS) hindfoot scores [17] at the 24-month follow-up. The postoperative wound-related complications were also recorded.

\section{Radiological evaluation}

Lateral and axial radiographs and CT scans were obtained immediately post-operation to assess the reduction of the articular surface and the fracture fixation. Physical examination and lateral and axial radiographs of the injured foot were performed at each follow-up evaluation. The calcaneal anatomical parameters, including Böhlers angle, Gissanes angle, height, width, and length were measured by radiographs or CT scans postoperatively and at the final follow up. Hardware was removed from each patient at 12 months postoperatively, unless the patient had indications for earlier hardware removal.

\section{Statistical analysis}

Statistical analysis was performed with SPSS 17.0 software for Windows. Continuous data with a normal distribution were expressed as the mean \pm standard deviation. The Mann-Whitney $U$ test and non-paired t test were used to compare differences continuous variables with non-normal distributions and approximately normally distributed respectively. Categorical data were statistically analyzed by Chi-square test or Fisher's exact test $(n<40$ or $t<1)$. A $p$ value of $<0.05$ was considered statistically significant.

\section{Results}

A total of 146 adult patients were assessed in our study (Fig. 3). Thirty-two patients did not meet the inclusion criteria and were excluded. Eighteen patients were excluded due to severe medical ailments (4 patients) and non-adherence to the treatment plan (14 patients). Therefore, 96 patients with an average age of 40.3 years old (range, 18 to 66 years) including 80 men and 16 women participated in the study. 16 patients were lost during the follow-up. A remaining of 80 patients (66 men and 14 women) were followed-up for 24 months averagely (range, 20 to 29 months).

Forty-two patients were treated with percutaneous reduction, cannulated screw fixation and CSC grafting $(\mathrm{PR}+\mathrm{CSC}$ group), and 38 patients were treated with 

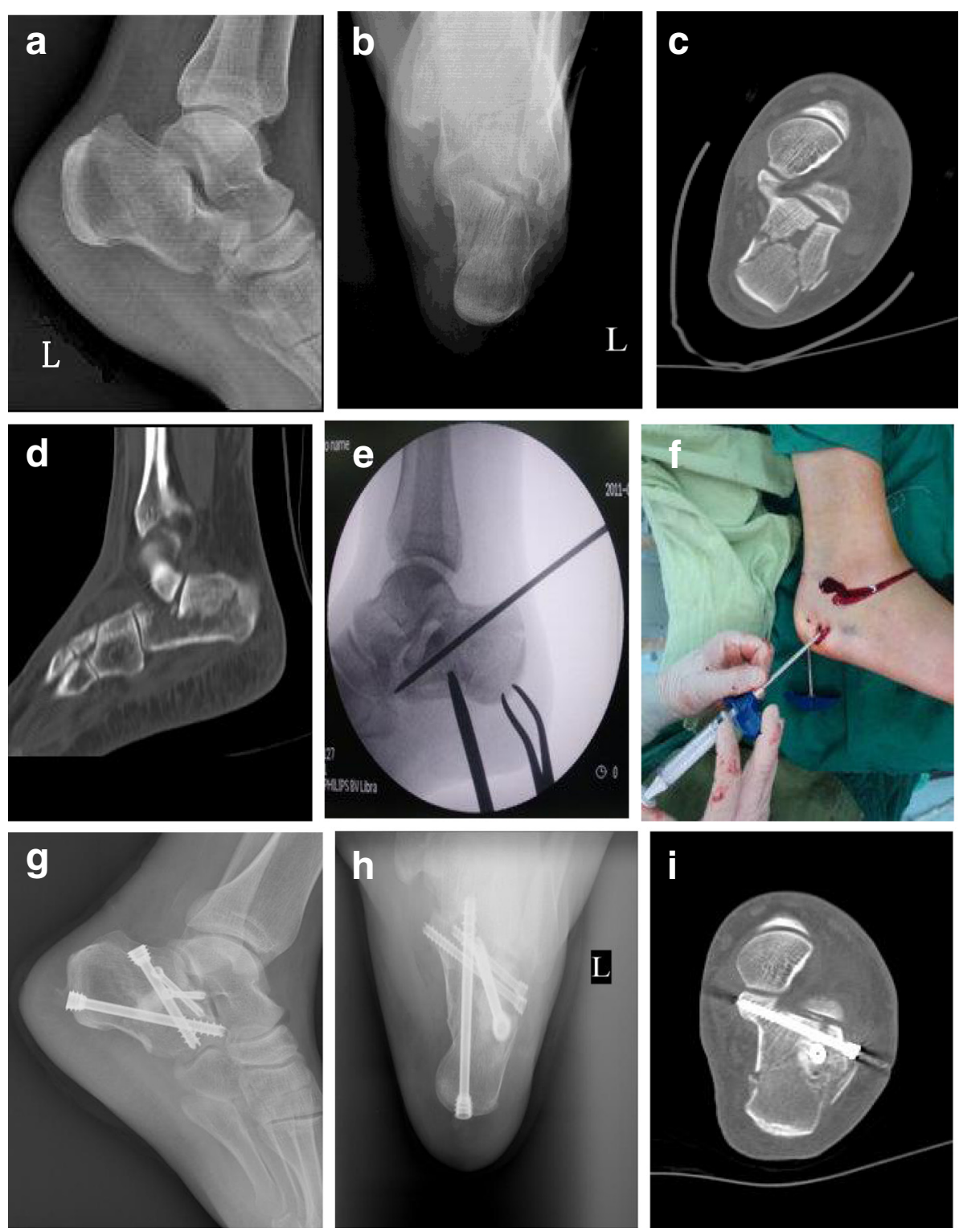

Fig. 1 A 42-year-old female patient suffered left lateral displaced intra-articular calcaneal fracture caused by a fall from a height. Preoperative X-ray: lateral view (a) and axial view (b) showed significantly decreased calcaneal height/length, Bohler angle, Gissanes angle, and significantly increased calcaneal width. A preoperative CT-scan (c, d) showed a Sanders Type-II. A calcaneal fracture with collapse of the subtalar articular surface. Intraoperative fluoroscopy (e) showed the reduction of the subtalar joint surface through two Schanz pins that were used to lever and percutaneously push up the depression of the subtalar joint surface. The CSC was injected into the void of the body (f). Postoperative X-ray: lateral view $(\mathbf{g})$ and axial view $(\mathrm{H})$ showed calcaneal anatomical reduction. Postoperative CT-scan (i) showed the reduction of the articular surface was satisfactory, however, the restoration of calcaneal width was still dissatisfactory

the minimally invasive sinus tarsi approach and plate fixation (MISTA group). In the PR+CSC group, there were 34 men and 8 women with a mean age of 39.5 years (range, 18 to 66 years). The affected side of foot included 22 right feet and 20 left feet. According to the Sanders classification, there were 32 Type-II fractures and 10 Type-III fractures. Fall from height (30 fractures) were the primary injury mechanisms, besides motorvehicle accident ( 8 fractures), and others (5 fractures). The
MISTA group consisted of 32 men and 6 women with an average age of 40.7 years (range, 18 to 65 years). There were 24 right feet injury and 14 left feet injury with 30 Type-II fractures and 8 Type-III fractures. Similarly, the injury mechanisms were also fall from height (30 fractures), motor-vehicle accident (6 fractures), and others ( 2 fractures; Table 1). There were no significant differences between the two groups regarding sex, age, fracture classification, affected side of foot and injury 

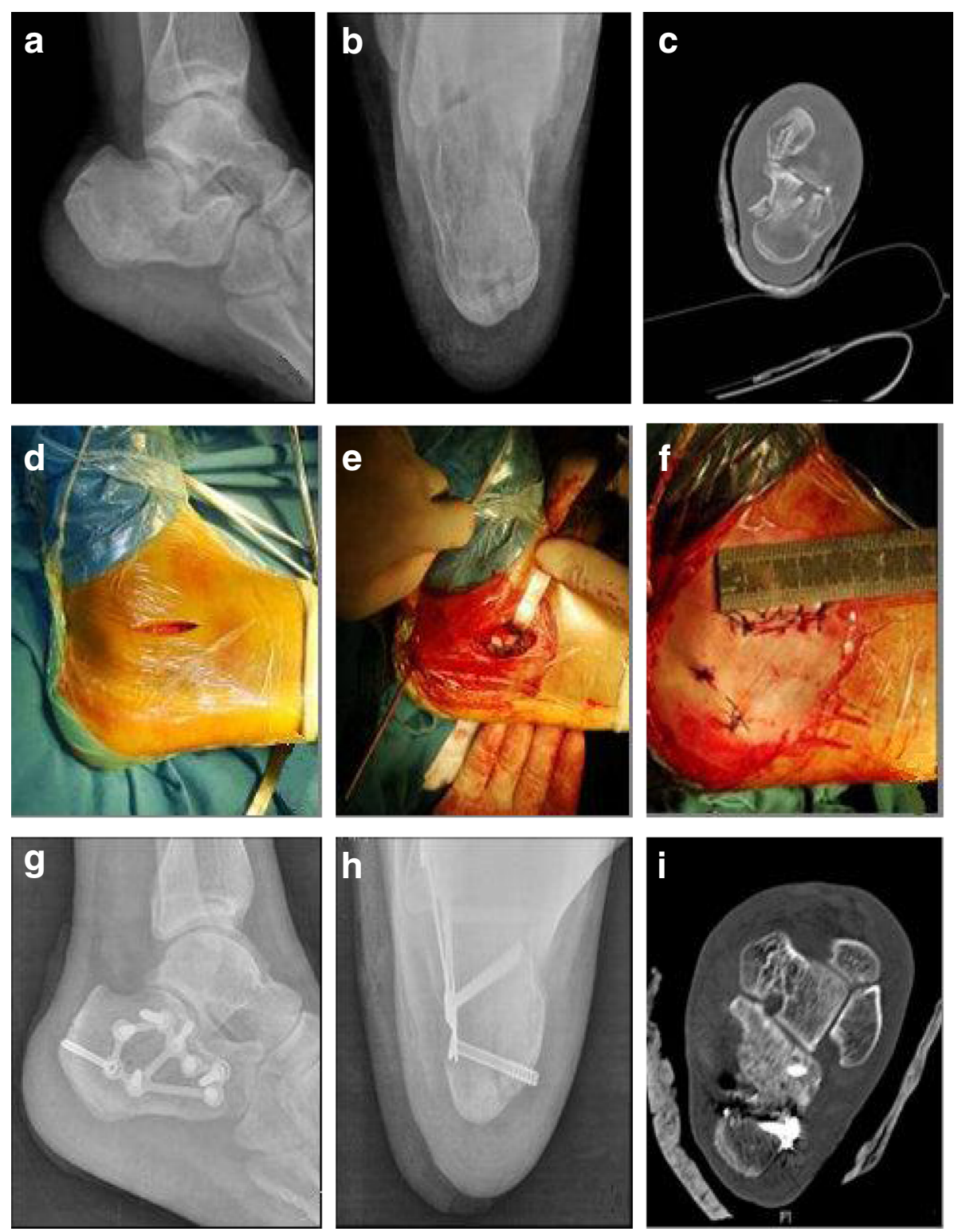

Fig. 2 A 38-year-old male patient suffered right lateral displaced intra-articular calcaneal fracture caused by a fall from a height. Preoperative X-ray: lateral view (a) and axial view (b) showed the calcaneal height/length, Bohler angle, and the Gissanes angle was significantly decreased, and the calcaneal width was significantly increased. Preoperative CT-scan (c) showed a Sanders Type-IIIAB calcaneal fracture with collapse of the subtalar articular surface. Intraoperative incision showed a $4.0 \mathrm{~cm}$ minimally invasive sinus tarsi approach (d), the anatomical plate was placed through the subcutaneous tunnel (e), and the incision was sutured (f). Postoperative X-ray: lateral view (g) and axial view (h) showed calcaneal anatomical reduction. Postoperative CT-scan (i) showed anatomic reduction of the articular surface and restoration of the calcaneal width

mechanisms. However, the average time from initial injury to operation was 2.9 days in the PR+CSC group, which was significantly shorter compared with 4.3 days in the MISTA group $(p<0.001)$. The average operation time was also significantly shorter in the $\mathrm{PR}+\mathrm{CSC}$ group than that in the MISTA group (39.7 min vs $64.2 \mathrm{~min}, p<0.001$; Table 1 ).

As shown in Table 2, according to the AOFAS scoring system, 22 fractures $(52.4 \%)$ were assessed as excellent; $12(28.6 \%)$ as good; $6(14.3 \%)$ as fair; and $2(4.8 \%)$ as poor in the PR+CSC group. In the MISTA group, an excellent, good, fair, or poor result was achieved in 20 (52.6\%), 14 (36.8\%), 3 (7.9\%), and 1 (2.6\%) fracture, respectively. The mean overall AOFAS score was not significantly different between the two groups (PR + CSC group: $84.6 \pm 6.6$ vs MISTA group: $82.5 \pm 5.7$ ). Patients in the PR+CSC group had better outcomes on all six variables than patients in the MISTA group. However, there were significant differences between the two groups with regard to the variable of sagittal 


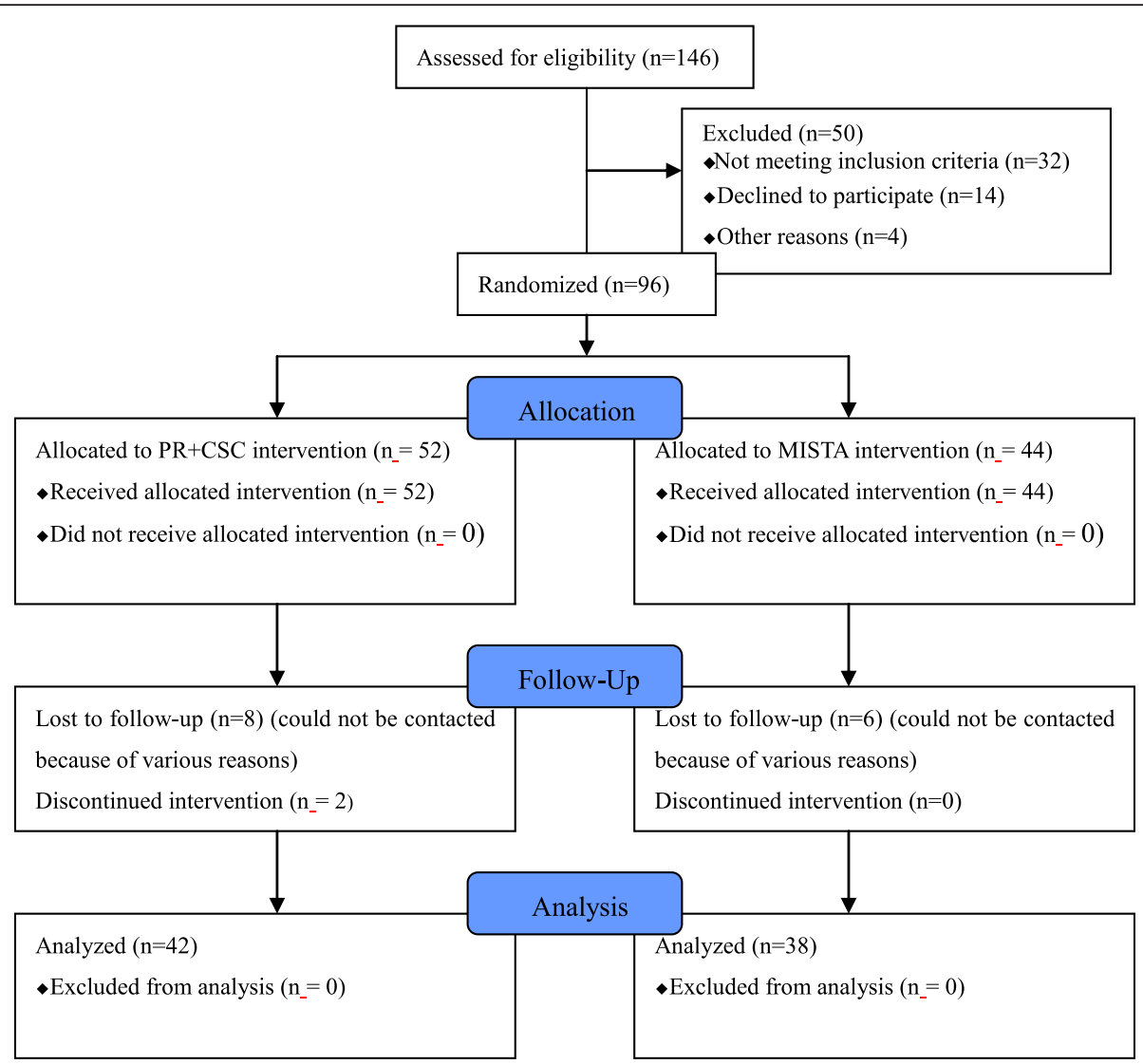

Fig. 3 Consolidated Standards of Reporting Trials (CONSORT) 2010 flow diagram depicting fracture allotment in both groups

Table 1 Comparison of the general characteristics of the two

\begin{tabular}{|c|c|c|c|}
\hline \multirow[t]{2}{*}{ General information } & \multirow{2}{*}{$\begin{array}{l}\text { PR+CSC Group } \\
N=42\end{array}$} & \multirow{2}{*}{$\begin{array}{l}\text { MISTA Group } \\
N=38\end{array}$} & \multirow[t]{2}{*}{$P$ Value } \\
\hline & & & \\
\hline Age (yr) & $39.5 \pm 10.5$ & $40.7 \pm 10.3$ & 0.642 \\
\hline Sex & & & 0.702 \\
\hline Male & 34 & 32 & \\
\hline Female & 8 & 6 & \\
\hline Sanders classification & & & 0.768 \\
\hline Type-II & 32 & 30 & \\
\hline Type-III & 10 & 8 & \\
\hline Side of injured & & & 0.330 \\
\hline Right & 22 & 24 & \\
\hline Left & 20 & 14 & \\
\hline Injury mechanism & & & 0.754 \\
\hline Falling accident & 30 & 30 & \\
\hline Traffic accident & 8 & 6 & \\
\hline Other causes & 4 & 2 & \\
\hline Time to operation (days) & $2.9 \pm 1.1$ & $4.3 \pm 1.4$ & $<0.001$ \\
\hline Operation time (min) & $39.7 \pm 7.6$ & $64.2 \pm 8.6$ & $<0.001$ \\
\hline
\end{tabular}

motion and hindfoot motion in the AOFAS scoring system $(p<0.05 ;)$.

Among the patients who achieved good or excellent results, there were 32 Type-II fractures and 2 Type-III fractures in the $\mathrm{PR}+\mathrm{CSC}$ group. While in the MISTA group, there were 28 Type-II fractures and 6 Type-III fractures. The good to excellent rate of the patients with Sanders Type-II and Sanders Type-(II + III) fractures between the two groups showed no significant differences. However, only for Sanders Type-III fractures, the good to excellent rate in the $\mathrm{PR}+\mathrm{CSC}$ group was significantly lower than that in the MISTA group (20 vs $75 \%$; $p<0.05$; Table 3 ).

Eight patients developed postoperative wound-healing complications, including superficial surgical site infections ( 1 patient in the $\mathrm{PR}+\mathrm{CSC}$ group and 3 patients in the MISTA group), deep surgical site infections (2 patients in the MISTA group), hematoma and wound-edge necrosis (1 patient each in the MISTA group) (Table 4). The superficial surgical site infections and wound hematoma were resolved by dressing changes. The deep surgical site infections, in contrast, involved the hardware, which required hardware removal. The one wound-edge necrosis case was cured by debriding twice 
Table 2 The functional outcome according to AOFAS scores for the two groups

\begin{tabular}{llll}
\hline & $\begin{array}{l}\text { PR+CSC Group } \\
(\mathrm{x} \pm \mathrm{s})\end{array}$ & $\begin{array}{l}\text { MISTA Group } \\
(\mathrm{x} \pm \mathrm{s})\end{array}$ & $P$ Value \\
\hline Pain & $34.0 \pm 6.3$ & $33.2 \pm 6.2$ & 0.526 \\
Activity limitation & $8.4 \pm 1.9$ & $8.2 \pm 1.9$ & 0.686 \\
Walking surface & $3.9 \pm 1.5$ & $3.8 \pm 1.3$ & 0.784 \\
Gait abnormality & $6.0 \pm 2.5$ & $5.8 \pm 2.4$ & 0.447 \\
$\begin{array}{l}\text { Sagittal motion } \\
\text { (flexion plus extension) }\end{array}$ & $7.0 \pm 1.8$ & $6.0 \pm 2.2$ & 0.037 \\
$\begin{array}{l}\text { Hindfoot motion } \\
\text { (inversion plus eversion) }\end{array}$ & $5.3 \pm 1.3$ & $4.5 \pm 1.7$ & 0.021 \\
Total & $84.6 \pm 6.6$ & $82.5 \pm 5.7$ & 1.131 \\
\hline
\end{tabular}

and dressing changes. Sural nerve damage was found in two patients in the MISTA group. Musculus peroneus brevis injury was reported in two patients respectively in the two groups, which were sutured immediately intraoperatively and immobilized by plaster for four weeks postoperatively. Overall, the wound-related complication rate was $7.1 \%$ in the $\mathrm{PR}+\mathrm{CSC}$ group and $28.9 \%$ in the MISTA group, the difference being statistically significant $(p=0.010)$. Two patients had early removal of the implant due to deep surgical site infections, and the other 78 patients had the hardware removed around 12-month post-surgery.

We did not find significant differences in calcaneal Böhlers angle, Gissanes angle, and the height and length between the two groups preoperatively, immediately postoperatively and at the final follow-up. The calcaneal width between the two groups preoperatively also showed no significant difference. However, the calcaneal width immediately postoperatively and at the final follow-up in the MISTA group had obviously improved compared to those in the PR+CSC group $(p<0.001$; Table 5$)$

The mean time from surgery to partial weight-bearing was comparable between the $\mathrm{PR}+\mathrm{CSC}$ group and the MISTA group (6.2 weeks vs 6.0 weeks; $p<0.05$ ). At the last follow-up, posttraumatic subtalar arthritis was observed in two patients in the PR+CSC group and one patient in the MISTA group as shown by radiographs.

Table 3 Good to excellent results in the two groups according to the Sanders classification

\begin{tabular}{llll}
\hline $\begin{array}{l}\text { Sanders } \\
\text { classification }\end{array}$ & $\begin{array}{l}\text { Type-II } \\
\text { no }(\%)\end{array}$ & $\begin{array}{l}\text { Type-III } \\
\text { no }(\%)\end{array}$ & $\begin{array}{l}\text { Type (II+ III) } \\
\text { no (\%) }\end{array}$ \\
\hline PR+CSC Group & $32(100 \%)$ & $2(20 \%)$ & $34(81.0 \%)$ \\
MISTA Group & $28(93.3 \%)$ & $6(75 \%)$ & $34(89.5 \%)$ \\
$P$ Value & 0.230 & 0.020 & 0.286 \\
\hline
\end{tabular}

Table 4 Comparison of the postoperative wound-related complications in the two groups

\begin{tabular}{llll}
\hline Complications & $\begin{array}{l}\text { PR+CSC Group } \\
\text { no (\%) }\end{array}$ & $\begin{array}{l}\text { MISTA Group } \\
\text { no (\%) }\end{array}$ & P Value \\
\hline Wound-healing complications & $1(2.4 \%)$ & $7(18.4 \%)$ & 0.024 \\
$\quad 1$ & 3 & \\
Superficial infection & 1 & 2 & \\
Deep infection & 0 & 1 & \\
Hematoma & 0 & 1 & \\
Wound-edge necrosis & 0 & $2(5.3 \%)$ & 0.222 \\
Sural nerve injury & 0 & $2(5.3 \%)$ & 1.000 \\
$\begin{array}{l}\text { Musculus peroneus brevis } \\
\text { injury }\end{array}$ & $2(4.7 \%)$ & $11(28.9 \%)$ & 0.010 \\
Total & $3(7.1 \%)$ & & \\
\hline
\end{tabular}

\section{Discussion}

The optimal management of displaced intra-articular calcaneal fractures remains controversial [1]. An increasing number of studies have shown a trend toward better functional outcomes in the operatively managed groups than those treated non-operatively [18, 19]. However, the high concern about wound-related complications with the extensile lateral L-shaped approach has troubled many orthopedic surgeons [7]. The complication rate after surgical treatment has been reported to range

Table 5 Radiographic results for the two groups

\begin{tabular}{llll}
\hline & $\begin{array}{l}\text { PR+CSC Group } \\
(\mathrm{x} \pm \mathrm{s})\end{array}$ & $\begin{array}{l}\text { MISTA Group } \\
(\mathrm{x} \pm \mathrm{s})\end{array}$ & P Value \\
\hline Böhler angle (deg) & & & \\
Preop & $2.1 \pm 5.5$ & $2.2 \pm 7.0$ & 0.921 \\
Immediately Postop & $30.3 \pm 4.0$ & $30.4 \pm 3.3$ & 0.920 \\
Final follow-up & $28.6 \pm 3.6$ & $29.2 \pm 3.5$ & 0.425 \\
Gissanes angle (deg) & & & \\
Preop & $93.5 \pm 7.4$ & $93.3 \pm 7.5$ & 0.866 \\
Immediately Postop & $120.2 \pm 7.2$ & $119.9 \pm 6.0$ & 0.830 \\
Final follow-up & $117.1 \pm 6.8$ & $117.6 \pm 6.1$ & 0.724 \\
Calcaneal width (mm) & & & \\
Preop & $39.8 \pm 2.6$ & $39.1 \pm 2.5$ & 0.251 \\
Immediately Postop & $34.7 \pm 2.2$ & $33.0 \pm 1.8$ & $<0.001$ \\
Final follow-up & $35.3 \pm 2.4$ & $33.4 \pm 1.9$ & $<0.001$ \\
Calcaneal height (mm) & & & \\
Preop & $31.4 \pm 4.2$ & $30.9 \pm 3.6$ & 0.603 \\
Immediately Postop & $40.3 \pm 5.4$ & $40.6 \pm 4.6$ & 0.722 \\
Final follow-up & $38.7 \pm 2.7$ & $39.3 \pm 3.1$ & 0.318 \\
Calcaneal length (mm) & & $68.2 \pm 2.6$ & 0.059 \\
\hline Preop. & $63.5 \pm 2.1$ & $63.2 \pm 2.8$ & 0.543 \\
Immediately Postop & $67.9 \pm 4.3$ & $69.1 \pm 2.7$ & 0.157 \\
Final follow-up & $66.9 \pm 3.9$ & $68.2 \pm$ & \\
\hline
\end{tabular}


from 11 to $25 \%[4-7,20,21]$. As one of the most widely applied minimally invasive techniques, the sinus tarsi approach has the advantage of direct visualization of the posterior articular facet and fewer wound-related complications $[11,12,15]$. In order to minimize the woundrelated complications and explore the optimal treatment for DIACFs, in 2006, we introduced percutaneous reduction, cannulated screw fixation and CSC grafting for treatment of DIACFs. We reported the first clinical data using this technique to treat 90 patients with 90 DIACFs in an article published in 2011 [16]. We found that, compared with ORIF, this minimally invasive technique allowed earlier weight bearing, reduced subtalar joint stiffness, and improved patient satisfaction. To further optimize the minimally invasive percutaneous technique in the treatment of DIACFs, in this study, we aimed to compare the functional outcomes, radiographic results and the postoperative wound-related complications between the minimally invasive percutaneous fixation and the minimally invasive sinus tarsi approach.

The assessment of functional outcomes in our study revealed an overall good to excellent rate of $81.0 \%$ in the PR+CSC group compared with $89.5 \%$ in the MISTA group. The mean AOFAS score was 84.6 in the PR+CSC group, which was higher than that 82.5 in the MISTA group. However, both differences were not statistically significant. There was also no significant difference between the two groups in terms of the good to excellent rate for Sanders Type-II fractures. Wang YM et al. reported percutaneous reduction and Steinman pin fixation minimized complications and achieved functional outcomes comparable to those of the open techniques in patients with Sanders Type-II calcaneal fractures [22]. For Sanders Type-III fractures, however, the MISTA group could achieve better functional outcomes than the $\mathrm{PR}+\mathrm{CSC}$ group. For this reason, we believed that the minimally invasive sinus tarsi approach was superior to the percutaneous cannulated screw fixation in acquiring a better articular surface reduction, especially for Sanders Type-III fractures. Thus different functional outcomes may be attributed to different qualities of articular reduction. Consistently, Mulcahy et al. also suggessted that a minor residual step-off of the posterior facet could cause a significant load shift within the subtalar joint [23], which might have an adverse effect on functional outcome $[24,25]$. Therefore, using the percutaneous reduction and cannulated screw fixation to treat comminuted calcaneal fractures may carry a risk of inadequate reduction of the articular surface.

The results analysis from the partial variables of the AOFAS scoring system suggested that patients in the PR + CSC group suffered from less pain, less activity limitations, less gait abnormality, and less demand on walking surfaces. Meanwhile, they obtained better sagittal motion and hindfoot motion than patients in the MISTA group. However, the difference was only statistically significant for two variables; sagittal motion and hindfoot motion, which suggests, compared with the minimally invasive sinus tarsi approach, that percutaneous reduction and cannulated screw fixation may be more minimally invasive and favor earlier and painless functional exercise.

The radiographic results revealed the significant differences between the two groups in terms of the calcaneal width immediately postoperatively and at the final follow-up. Therefore, the role of the percutaneous reduction and cannulated screw fixation restoring the calcaneal width was weaker than the minimally invasive sinus tarsi approach. Although it is known that the widened calcaneus is the main reason for patients suffering from lateral impingement syndrome [26-28], the difference in the overall functional outcomes between the two groups was not statistically significant. Our data suggested that the reduction of the posterior articular surface might be the most important factor associated with the functional outcome of calcaneal fractures [3, 24, 25].

The wound-healing complication rate was significantly lower in the PR+CSC group than that in the MISTA group (2.4 vs $18.4 \%$ ). The minimized disturbance to the blood supply and the soft tissue of the lateral aspect of the calcaneal [29], coupled with the significantly shorter operative time, may have contributed to the lower rate of wound-healing complications in the PR+CSC group. Furthermore, the PR+CSC group had a lower risk of sural nerve injury and musculus peroneus brevis injury compared with the MISTA group, however, the difference was not statistically significant. Although the overall complication rate was found to be quite high in the MISTA group, most of the complications appeared in the early days. The complication rate decreased significantly as our experience improved and the waiting time to operation extended.

There are a few limitations in this study. First, we had a relatively small number of patients and short average follow-up time. Therefore, further investigation with a larger sample size and longer follow-up time is needed to obtain more overall clinical data. In addition, the differences in surgeons' performances might have decreased the generalization power of this study. Assigning all the patients to only one surgeon would be much beneficial to reduce the variability caused by the surgeon's performance, but this would be practically impossible in our hospital. Furthermore, the quality of joint surface reduction was not specifically analyzed or compared between the two groups. It needs to be studied further.

\section{Conclusions}

The percutaneous reduction, cannulated screw fixation and CSC grafting for treatment of Sanders Type-II 
DIACFs can achieve almost equivalent functional outcomes compared with the minimally invasive sinus tarsi approach and plate fixation. The two techniques have their own advantages. The $\mathrm{PR}+\mathrm{CSC}$ grafting is superior to the MISTA procedure in terms of the average time between initial injury and operation, operation time, wound-related complications and subtalar joint activity. However, the MISTA procedure has its own advantages in improving the calcaneal width, providing clear visualization and more accurate reduction of the articular surface, especially for Sanders Type-III DIACFs. The functional outcomes of Sanders Type-III DIACFs treated by MISTA excelled the $\mathrm{PR}+\mathrm{CSC}$ grafting.

\section{Abbreviations}

AOFAS, American Orthopaedic Foot and Ankle Society; CONSORT, Consolidated Standards of Reporting Trials; CSC, calcium sulfate cement; CT, computed tomography; DIACFs, displaced intra-articular calcaneal fractures; MISTA, minimally invasive sinus tarsi approach; ORIF, open reduction and internal fixation; PR, percutaneous reduction

\section{Funding}

This research was supported by Zhejiang provincial medical and health technology project funding (No.2016KYA138) and Zhejiang provincial traditional chinese medicine scientific research fund project (No. 2016ZA141).

\section{Availability of data and materials}

The datasets supporting the conclusions of this article are included within the article.

\section{Authors' contributions}

YF, XS, JW, and $\mathrm{JH}$ initiated the study design. $\mathrm{JH}$ is the principal investigator. $Y Y$ is the programme coordinator. $Y Y$ conceived the intervention and, with the support of $X Y$, is responsible for execution of the intervention, recruitment of participants and the administration of the study. JK helps to coordinate data collection. LC and YF help with the recruitment and, together with JK, provide clinical expertise. All authors are ICH/GCP certified and contributed to the study protocol and approved the final manuscript.

\section{Competing interests}

The authors declare that they have no competing interest.

\section{Ethics and consent to participate}

All patients agreed to participate in this clinical trial by signing an informed consent form. The study was approved by the Ethical Board Review of the Second Affiliated Hospital of Wenzhou Medical University.

\section{CONSORT statement}

The manuscript was prepared according to CONSORT 2010 guidelines.

Received: 26 February 2016 Accepted: 9 June 2016

Published online: 15 July 2016

\section{References}

1. Rammelt S, Zwipp H. Calcaneus fractures: facts, controversies and recent developments. Injury. 2004;35:443-61.

2. Sanders R. Displaced intra-articular fractures of the calcaneus. J Bone Joint Surg Am. 2000;82:225-50.

3. Buckley $\mathrm{R}$, Tough $\mathrm{S}$, McCormack R, Pate G, Leighton R, Petrie $\mathrm{D}$, et al. Operative compared with nonoperative treatment of displaced intra- articular calcaneal fractures: a prospective, randomized, controlled multicenter trial. J Bone Joint Surg Am. 2002;84:1733-44.

4. Howard JL, Buckley R, McCormack R, Pate G, Leighton R, Petrie D, et al. Complications following management of displaced intra-articular calcaneal fractures: a prospective randomized trial comparing open reduction internal fixation with nonoperative management. J Orthop Trauma. 2003;17:241-9.

5. Koski A, Kuokkanen H, Tukiainen E. Postoperative wound complications after internal fixation of closed calcaneal fractures: a retrospective analysis of 126 consecutive patients with 148 fractures. Scand J Surg. 2005;94:243-5.

6. Cavadas PC, Landin L. Management of soft-tissue complications of the lateral approach for calcaneal fractures. Plast Reconstr Surg. 2007;120: 459-66. discussion 467-469.

7. Al-Mudhaffar M, Prasad CV, Mofidi A. Wound complications following operative fixation of calcaneal fractures. Injury. 2000:31:461-4.

8. Ramos RR, de Castro Filho CD, Ramos RR, Bittar CK, de Cillo MS, de Mattos CA, et al. Surgical treatment of intra-articular calcaneal fractures: description of a technique using an adjustable uniplanar external fixator. Strategies Trauma Limb Reconstr. 2014:9:163-6.

9. Rammelt S, Heineck J, Barthel S, Zwipp H. Percutaneous fixation of intraarticular calcaneus fractures. Techn Foot Ankle Surg. 2009:8:70-6.

10. Sampath Kumar V, Marimuthu K, Subramani S, Sharma V, Bera J, Kotwal P. Prospective randomized trial comparing open reduction and internal fixation with minimally invasive reduction and percutaneous fixation in managing displaced intra-articular calcaneal fractures. Int Orthop. 2014;38: 2505-12.

11. Schuberth JM, Cobb MD, Talarico RH. Minimally invasive arthroscopicassisted reduction with percutaneous fixation in the management of intra-articular calcaneal fractures: a review of 24 cases. J Foot Ankle Surg. 2009;48:315-22.

12. Hospodar P, Guzman C, Johnson P, Uhl R. Treatment of displaced calcaneus fractures using a minimally invasive sinus tarsi approach. Orthopedics. 2008; $31: 1112$.

13. Schepers T. The sinus tarsi approach in displaced intra-articular calcaneal fractures: a systematic review. Int Orthop. 2011;35:697-703.

14. Zhang T, Su Y, Chen W, Zhang Q, Wu Z, Zhang Y. Displaced intraarticular calcaneal fractures treated in a minimally invasive fashion: longitudinal approach versus sinus tarsi approach. J Bone Joint Surg Am. 2014:96:302-9.

15. Yeo JH, Cho HJ, Lee KB. Comparison of two surgical approaches for displaced intra-articular calcaneal fractures: sinus tarsi versus extensile lateral approach. BMC Musculoskelet Disord. 2015;16:63.

16. Chen L, Zhang G, Hong J, Lu X, Yuan W. Comparison of percutaneous screw fixation and calcium sulfate cement grafting versus open treatment of displaced intra-articular calcaneal fractures. Foot Ankle Int. 2011;32:979-85

17. Kitaoka HB, Alexander IJ, Adelaar RS, Nunley JA, Myerson MS, Sanders M Clinical rating systems for the ankle-hindfoot, midfoot, hallux, and lesser toes. Foot Ankle Int. 1994;15:349-53.

18. Radnay CS, Clare MP, Sanders RW. Subtalar fusion after displaced intraarticular calcaneal fractures: does initial operative treatment matter? J Bone Joint Surg. 2009;91:541-6.

19. De Boer AS, Van Lieshout EM, Den Hartog D, Weerts B, Verhofstad MH, Schepers T. Functional outcome and patient satisfaction after displaced intra-articular calcaneal fractures: a comparison among open, percutaneous, and nonoperative treatment. J Foot Ankle Surg. 2015;54:298-305.

20. Rak V, Ira D, Masek M. Operative treatment of intra-articular calcaneal fractures with calcaneal plates and its complications. Indian J Orthop. 2009:43:271-80.

21. Backes M, Schepers T, Beerekamp MS, Luitse JS, Goslings JC, Schep NW. Wound infections following open reduction and internal fixation of calcaneal fractures with an extended lateral approach. Int Orthop. 2014; 38:767-73.

22. Wang YM, Wei WF. Sanders II type calcaneal fractures: a retrospective trial of percutaneous versus operative treatment. Orthop Surg. 2015;7:31-6.

23. Mulcahy DM, McCormack DM, Stephens MM. Intra-articular calcaneal fractures: effect of open reduction and internal fixation on the contact characteristics of the subtalar joint. Foot Ankle Int. 1998;19:842-8.

24. Song KS, Kang CH, Min BW, Sohn GJ. Preoperative and postoperative evaluation of intra-articular fractures of the calcaneus based on computed tomography scanning. J Orthop Trauma. 1997;11:435-40. 
25. Gavlik JM, Rammelt S, Zwipp H. The use of subtalar arthroscopy in open reduction and internal fixation of intra-articular calcaneal fractures. Injury. 2002;33:63-71.

26. Chen W, Li X, Su Y, Zhang Q, Smith WR, Zhang X, et al. Peroneal tenography to evaluate lateral hindfoot pain after calcaneal fracture. Foot Ankle Int. 2011;32:789-95.

27. Schepers T, van Lieshout EM, van Ginhoven TM, Heetveld MJ, Patka P. Current concepts in the treatment of intra-articular calcaneal fractures: results of a nationwide survey. Int Orthop. 2008;32:711-5.

28. Wu Z, Su Y, Chen W, Zhang Q, Liu Y, Li M, et al. Functional outcome of displaced intra-articular calcaneal fractures: a comparison between open reduction/internal fixation and a minimally invasive approach featured an anatomical plate and compression bolts. J Trauma Acute Care Surg. 2012;73: 743-51.

29. Stein H, Rosen N, Lerner A, Kaufman H. Minimally invasive surgical techniques for the reconstruction of calcaneal fractures. Orthopedics. 2003; 26:1053-6.

Submit your next manuscript to BioMed Central and we will help you at every step:

- We accept pre-submission inquiries

- Our selector tool helps you to find the most relevant journal

- We provide round the clock customer support

- Convenient online submission

- Thorough peer review

- Inclusion in PubMed and all major indexing services

- Maximum visibility for your research

Submit your manuscript at www.biomedcentral.com/submit
Biomed Central 